\title{
Novel and economical apparatus to decrease direct droplet exposure during endoscopy in the ongoing COVID-19 pandemic: $A$ hypothesis-generating innovation
}

\author{
Abhinav Anand ${ }^{1} \cdot$ Ashish Agarwal $^{1} \cdot$ Deepak Gunjan $^{1} \cdot$ Anoop Saraya $^{1}$ (D \\ Published online: 3 July 2020 \\ (C) Indian Society of Gastroenterology 2020
}

The Corona Virus Disease (COVID)-19 pandemic has brought unprecedented challenges upon the health care system. Up to $20 \%$ of health care workers involved in the direct care of COVID-19 patients have been infected [1]. Of all the challenges faced by a gastroenterologist, endoscopy poses the maximum risk. Endoscopy is an aerosol-generating procedure with a significant, apparently unrecognized, droplet exposure [2]. Most guidelines [3-5] recommend deferring elective procedures, whereas pre-procedural screening, adequate usage of personal protective equipment, and strict infection control protocols are essential before emergency procedures. All protocols are dedicated to reducing exposure to droplet [6]; however, there is an unmet need in the ways to decrease the droplet expulsion at the patient level.

We have designed a novel apparatus made out of commonly available items to reduce direct droplet exposure to the endoscopist during various endoscopic pro-

Electronic supplementary material The online version of this article (https://doi.org/10.1007/s12664-020-01061-8) contains supplementary material, which is available to authorized users.

Anoop Saraya

ansaraya@yahoo.com

1 Department of Gastroenterology and Human Nutrition Unit, All India Institute of Medical Sciences, New Delhi 110 029, India cedures. Our apparatus consists of a transparent plastic sheet sandwiched between two surgical masks, with a hole in the center to accommodate the mouth guard, and a long camera cover sleeve attached to the front (Fig. 1). The mouth guard is then positioned in the mouth of the patient, and the apparatus completely covers the patient's face, including the mouth and the nose (Fig. 2). The ties of the masks are tied behind the patient to secure it in position. Endoscopy can then be carried out by inserting the endoscope through the distal end of the camera cover sleeve (Supplementary Video). The endoscopy assistant holds the distal end of the camera cover during the entire procedure and at the time of withdrawal of the endoscope, thus preventing any escape of droplets generated during the procedure. After removal of the endoscope, the mouth guard is removed and sterilized with glutaraldehyde for reuse, according to standard protocols. The remaining apparatus is discarded. The entire apparatus (excluding the mouth guard) costs approximately rupees (0.5\$).

This apparatus is economical and can be easily assembled using readily available items in the endoscopy suite. This is a hypothesis- generating innovation, and its efficacy to prevent the transmission of infection needs to be proven by appropriately designed studies before it can be used in practice. The clinical utility of the apparatus can be further explored in the current scenario, especially in the resource constrained settings. 
Fig. 1 The apparatus consisting of a mouth gag, surgical masks, a transparent sheet, and a camera cover as seen from the inner side (a) and the outer side (b) of the mouth guard

Fig. 2 The apparatus as applied on the patient
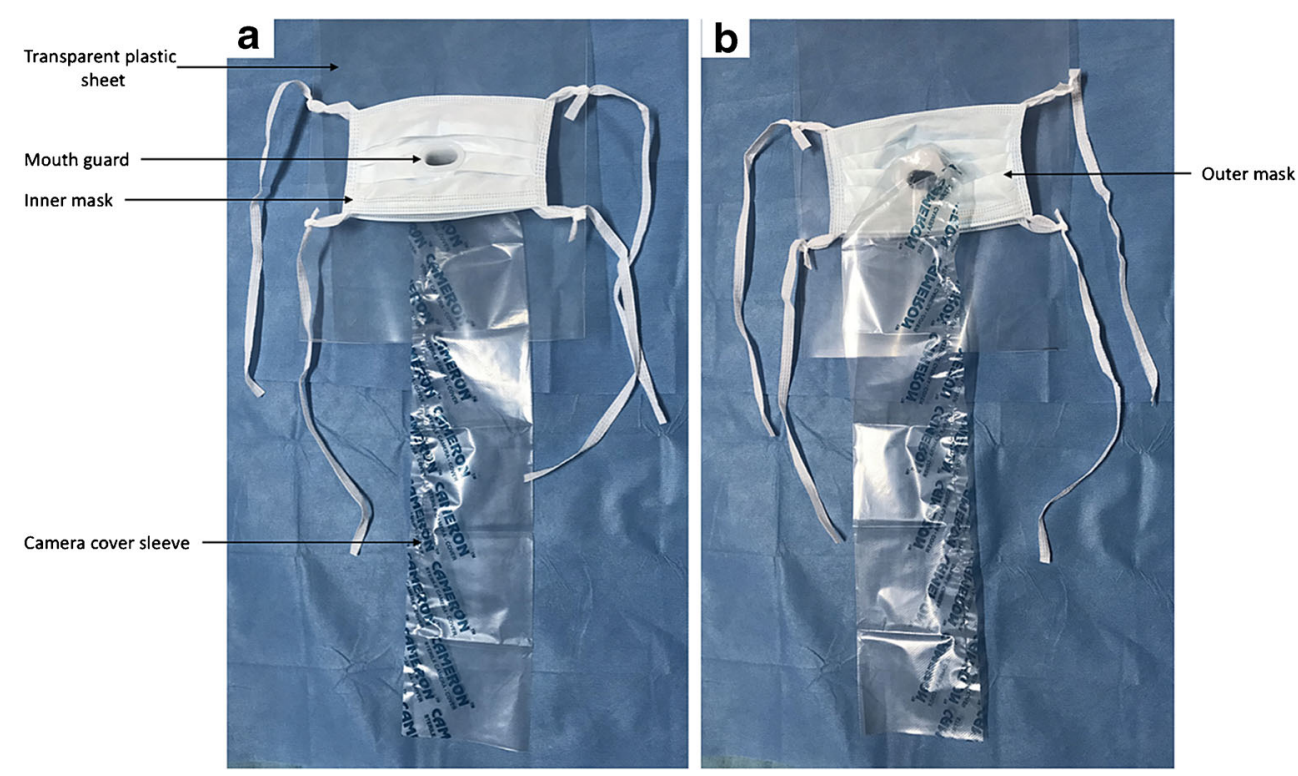

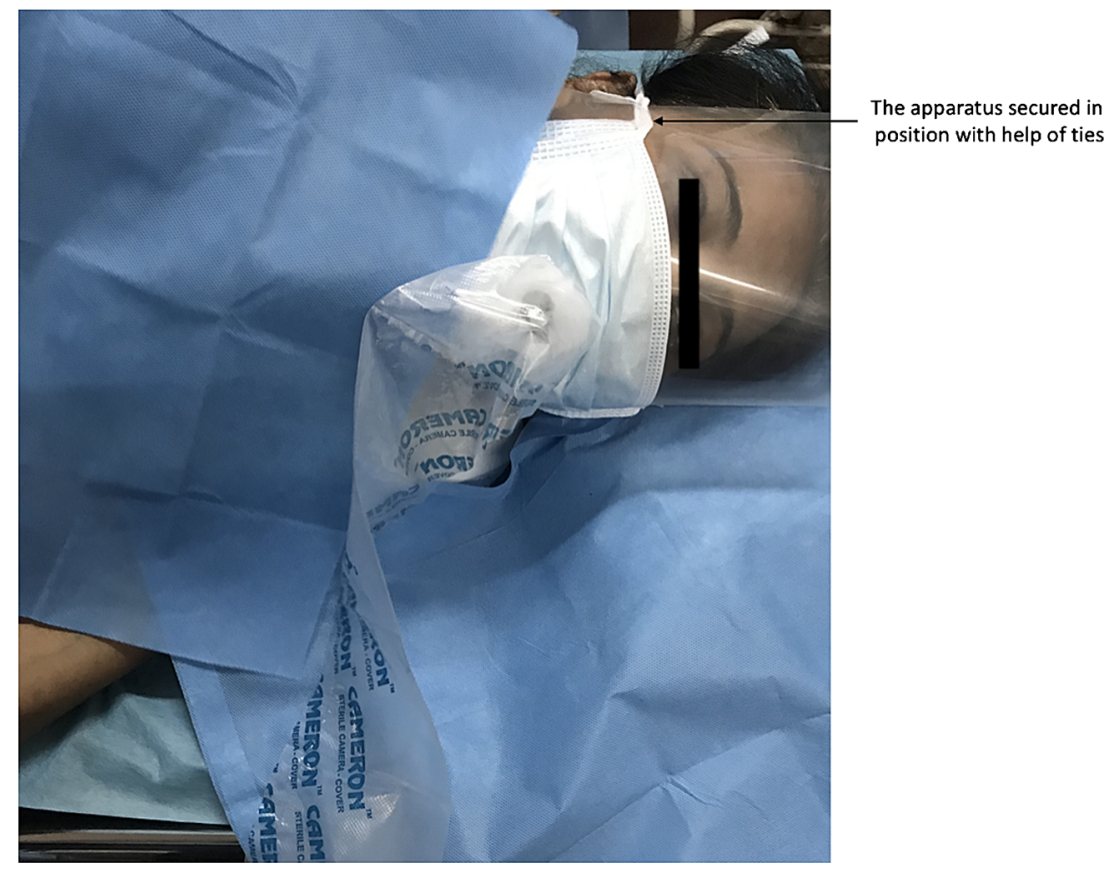




\section{Compliance with ethical standards}

Conflict of interest AA, AA, DG, and AS declare that they have no conflicts of interest.

Informed consent Obtained

Disclaimer The authors are solely responsible for the data and the contents of the paper. In no way, the Honorary Editor-in-Chief, Editorial Board Members, or the printer/publishers are responsible for the results/ findings and content of this article.

\section{References}

1. The Lancet. COVID-19: protecting health-care workers. Lancet. 2020;395:922.

2. Johnston ER, Habib-Bein N, Dueker JM, et al. Risk of bacterial exposure to the endoscopist's face during endoscopy. Gastrointest Endosc. 2019;89:818-24.
3. Chiu PWY, $\mathrm{Ng} \mathrm{SC}$, Inoue $\mathrm{H}$, et al. Practice of endoscopy during COVID-19 pandemic: position statements of the Asian Pacific Society for Digestive Endoscopy (APSDE-COVID statements). Gut. 2020;69:991-6.

4. British Society of Gastroenterology. Endoscopy activity and COVID-19: BSG and JAG guidance. Available: https://www.bsg. org.uk/covid-19-advice/endoscopy-activity-and-covid-19-bsg-andjag-guidance/.

5. American College of Gastroenterology Joint GI Society message on COVID 19. Available: https://gi.org/2020/03/15/joint-gi-societymessage-on-covid-19/.

6. Marchese M, Capannolo A, Lombardi L, Di Carlo M, Marinangeli F, Fusco P. Use of a modified ventilation mask to avoid aerosolizing spread of droplets for short endoscopic procedures during coronavirus COVID-19 outbreak. Gastrointest Endosc. 2020. https://doi.org/ 10.1016/j.gie.2020.03.3853 [Epub ahead of print].

Publisher's note Springer Nature remains neutral with regard to jurisdictional claims in published maps and institutional affiliations. 